NBER WORKING PAPER SERIES

INDEX-OPTION PRICING WITH

STOCHASTIC VOLATILITY AND THE

VALUE OF ACCURATE VARIANCE

FORECASTS

\author{
Robert F. Engle \\ Alex Kane \\ Jaesun Noh
}

Working Paper No. 4519
NATIONAL BUREAU OF ECONOMIC RESEARCH 1050 Massachusetts Avenue
Cambridge, MA 02138
November, 1993

This paper is part of NBER's research program in Asset Pricing. Any opinions expressed are those of the authors and not those of the National Bureau of Economic Research. 


\title{
INDEX-OPTION PRICING WITH \\ STOCHASTIC VOLATILITY AND THE \\ VALUE OF ACCURATE VARIANCE \\ FORECASTS
}

\section{ABSTRACT}

In pricing primary-market options and in making secondary markets, financial intermediaries depend on the quality of forecasts of the variance of the underlying assets. Hence, the gain from improved pricing of options would be a measure of the value of a forecast of underlying asset returns. NYSE index returns over the period of 1968-1991 are used to suggest that pricing index options of up to 90 -days maturity would be more accurate when: (1) using ARCH specifications in place of a moving average of squared returns; (2) using Hull and White's (1987) adjustment for stochastic variance in Black and Scholes's (1973) formula; (3) accounting explicitly for weekends and the slowdown of variance whenever the market is closed.

\author{
Robert F. Engle \\ Department of Economics \\ University of California, San Diego \\ San Diego, CA 92093-0508 \\ and NBER \\ Jaesun Noh \\ Department of Economics \\ University of California, San Diego \\ San Diego, CA 92093-0508
}
Alex Kane
Graduate School of International and Pacific Studies
University of California, San Diego
San Diego, CA 92093-0508




\section{Introduction}

When distributions of asset returns are time varying, forecasts of asset-return variance are valuable. Even passive investors need periodic variance forecasts to calibrate asset allocation. More critically, financial intermediaries who must offer competitive bids on primary-market options, or make market in outstanding options, need continuous updating of variance forecasts.

Since the variance of the underlying asset return is the only unobserved variable in the Black and Scholes's (1973) valuation formula for options, ${ }^{1}$ updates of variance are the major contestable input to valuation of contingent contracts by direct parties or financial intermediaries.

Emphasis on improved variance forecasts also calls for a corresponding adaptation of the pricing model that will explicitly account for stochastic volatility. When variance risk is not priced, ${ }^{2}$ and the return distribution can be well approximated by a diffusion process, the Hull and White's (1987) model is acceptable. Operationally, we must average the would be Black-Scholes prices for all possible variance rates over the life of the option, using a variance forecasting algorithm to generate the probability distribution of future variance rates. Another nuisance with stochastic volatility is that we cannot invert observed option prices to obtain the correct implied volatility - a widely used practice since Schmalensee and Trippi (1978).

A natural criterion for choosing between any pair of competing methods to forecast

\footnotetext{
${ }^{1}$ However, as Brenner and Galai (1986) observe, it is not all that clear which asset should be observed to obtain the risk-free rate in the Black-Scholes formula.

${ }^{2}$ The volatility of the variance of an underlying asset will not be priced if it is non-systematic. When the variance is priced, the Black-Scholes formula in the Bull and White's (1987) model must be amended to account for a deficiency in the asset risk premium. See McDonald and Siegel (1984) and Heston (1992).
} 
the variance of the rate of return on an asset would be the expected incremental profit from replacing the lesser forecast with the better one. We used this principle in Engle, Hong, Kane and Noh (1993) to estimate the profit from improving variance forecasts in pricing one-day index options (see also an application by Muller (1991) at BARRA), and reported that the evidence favored GARCH forecasts. ${ }^{3}$ Obviously, implications that apply to trading options of only one day maturity have little practical use. The role of variance forecasts in pricing options of longer maturity is the focus of this paper.

When daily variance forecasts are used to price options of longer maturities, the persistence of changes in the variance rate affects the economics of pricing options in two ways: first, the greater the volatility of the variance rate over the life of the option, the more acute the need for an option-pricing model that accounts for stochastic volatility. Moreover, a significant correlation of changes in the variance rate with economic factors will worsen the accuracy of the Black-Scholes model, and reduce the remedial potential of the Hull-White adjustment. Second, the greater the volatility and persistence of changes in the variance rate, the greater the benefits from using one-day forecasts for longer-maturity options. Put another way, if changes in the variance do not persist, then the value of one-day forecasts for longer horizons will deteriorate quickly.

In this paper we measure the economic value of two innovations in forecasting daily variance rates: first, we use GARCH forecasts as an alternative to a moving average of squared returns; second, we introduce a weekend calendar variable to the estimation and forecasting algorithm to account for how long the market is closed, and the degree of

\footnotetext{
${ }^{3}$ We compared GARCH $(1,1)$ to three versions of a moving average of squared residuals. Each forecast algorithm was used to provide a rolling daily forecast of the NYSE-index return variance. We used three alternative lengths of past observations to estimate the parameters: 300 days, 1,000 days and 5,000 days. The twelve time series of forecasts were used to price and hypothetically trade one-day options. The GARCE forecasts were decidedly superior in the NYSE sample for $1962-1990$. Using an estimation interval of 1,000 days was slightly better than either 300 or 5,000 days.
} 
slow-down of the variance rate during the time the market is closed. We also measure the value of using the Hull-White algorithm with the Black-Scholes option-pricing model to account for stochastic volatility in conjunction with the improved variance models.

In section 2 we describe the variance forecasts for the NYSE index that are used in the exercise. In Section 3 we use Hansen and Hodrick's (1980) method to compute volatility of overlapping return series. We also compute critical values to test the significance of mean estimators of the return series for fixed ratios of overlapping period to sample period. In Section 4 we use the return history of the NYSE index over the period, 1968 - 1991, to determine the realized payoffs to hypothetical options of various maturities on the NYSE index. We use these payoffs to examine the profitability of varjous index-option trading strategies related to variance forecasts. Section 5 concludes the paper.

\section{The Variance-Forecast Algorithms}

Engle, Hong, Kane and Noh (1993) used twelve different specifications to generate alternative daily forecasts for the variance of the returns on the NYSE index and used these forecasts to price one-day options on the NYSE index. They operated a simulated market in these options as follows. Each day, differences in the variance forecasts of the twelve algorithms resulted in different reservation prices for one-day options on the underlying index. These differences triggered trades in the options among twelve hypothetical agents, each using one of the forecast algorithms. An agent with a higher variance forecast had a higher reservation price for the one-day option. Hence this agent would buy a straddle (one call and one put option) on a $\$ 1$ share of the NYSE index from any of the other eleven agents with lower forecast/reservation prices. The exercise 
price for these straddles was set at $\$ 1$ plus the risk-free rate, and a trade between two agents was executed at the average of the reservation prices of the two agents, that is, at the average of the bid/ask prices. Upon expiration of each one-day straddle over the period 1962 - 1990, the realized daily returns on the NYSE were used to compute the profit from each daily trade between each pair of agents. Overall, ARCH models showed the highest profits and smallest standard deviations. A moving-average of 300 squared residuals was the nearest competitor to ARCH forecasts.

In this paper we extend the setting by supposing that on every day, at the money options, straddles are traded with maturities from one day to one year. Positions are held to maturity when profits are revealed. Consequently, traders must forecast volatilities up to one year ahead, and account for uncertain volatility in pricing straddles. We consider several models. We use the 300 day moving-average as a simple variance estimate for all horizons. Of the ARCH family of specifications first introduced by Engle (1982) and surveyed in Bollerslev, Chou, and Kroner (1992), we use the $\operatorname{GARCH}(1,1)$ specification for the NYSE return series:

$$
\begin{aligned}
& R_{t}=a_{0}+\epsilon_{t} \\
& h_{t}=b_{0}+b_{1} \epsilon_{t-1}^{2}+b_{2} h_{t-1} .
\end{aligned}
$$

In order to generate variance forecasts of horizons from one day up to $T$ days, the most recent 1,000 daily observations are first used to estimate the parameters $a_{0}, b_{0}, b_{1}, b_{2}$ in equation (1). The forward volatility forecasts are then computed from: 


$$
\begin{aligned}
h_{t, t+1} & =b_{0}+b_{1} \epsilon_{t}^{2}+b_{2} h_{t} \\
h_{t, t+k} & =b_{0}+b_{1} E\left[\epsilon_{t+k-1}^{2} \mid \omega_{t}\right]+b_{2} h_{t, t+k-1} \\
& =b_{0}+\left(b_{1}+b_{2}\right) h_{t, t+k-1}, k=2, \ldots, T
\end{aligned}
$$

where $h_{t, q+k}$ is the prediction of $h_{q+k}$ at time $t$. The average volatility is $(1 / T) \sum_{k=1}^{T} h_{t, t+k}$. We denote this forecast as GARCH.

We expect the mean and variance of the return from market close to close to be affected by calendar time, that is, mean and variance from Friday close to Monday close are expected to be greater than, say, from Wednesday close to Thursday close, and more so for long weekends. However, French and Roll (1986) showed that the variance rate slows down significantly in days when the market is closed. In an attempt to capture this phenomenon, we modify (1) and (2) multiplicatively as follows.

$$
\begin{aligned}
h_{t} & =d_{t}^{\delta}\left[b_{0}+d_{t-1}^{-\delta}\left(b_{1} \epsilon_{t-1}^{2}+b_{2} h_{t-1}\right)\right] \\
h_{t, t+1} & =d_{t+1}^{\delta}\left[b_{0}+d_{t}^{-\delta}\left(b_{1} \epsilon_{t}^{2}+b_{2} h_{t}\right)\right] \\
h_{t, t+k} & =d_{t+k}^{\delta}\left\{b_{0}+d_{i \div k-1}^{-s}\left[b_{1} E\left(\epsilon_{t+k-1}^{2} \mid \omega_{t}\right)+b_{2} h_{t, t+k-1}\right]\right\} \\
& =d_{t+k}^{\delta}\left\{b_{0}+d_{t ; k-1}^{-s}\left[\left(b_{1}+b_{2}\right) h_{t, t+k-1}\right]\right\}, k=2, \ldots, T,
\end{aligned}
$$

where $d_{t}$ is a calendar days variable that gives the number of calendar days between the close of the previous trading day $(t-1)$ and the close of the trading day $t$. As explained below, $d_{t}$ in the variance equation is raised to the power $\delta$ which measures the average 
speed of the variance rate over the $d_{t}$ calendar days of trading day $t$. As before, $h_{t, t+k}$ is the variance forecast made at date $t$ for date $t+k$. We denote the GARCH forecast adjusted for market closures by GARCH-D.

Assuming that variance surprises are independent of economic factors, we can use the Hul-White modification to the Black-Scholes formula. In order to test the potential economic value of the Hull-White modification we use (2) as follows. For each date $t+k ; k=1, \ldots, T$, we generate 1,000 sets of $\epsilon_{i+k}(j) ; j=1, \ldots, 1000$, from a standard normal distribution. For each $j$ we calculate the average daily variance rate over the $k=1, \ldots, T$ days to maturity of the option, and use this variance rate to calculate a price which is contingent on the $j$-th path. The average of these prices (over $j=$ $1, \ldots, 1000$ iterations) is the Hull and White's (1987) modified price. We denote these integrated prices by GARCH-I. A forecast that uses weekend dummies throughout the integration will be denoted GARCH-DI.

\section{Computation of Volatility of Overlapping Expected re- turn series}

To show that one variance forecast method outperforms other methods significantly; we need to compute standard errors of the mean of the return series to employ a $t$-test. Hansen and Hodrick (1980) suggested a method to compute standard errors for overlapping observations. Recently, Richardson and Stock (1989) proposed a different inference based on a novel asymptotic argument. The difference is whether we treat multi-day return series as stationary processes or non-stationary processes.

Let $R_{m, t}$ be the one day return from options trading, which is assumed to be unpre- 
dictable from past values, except for a constant $\mu$. That is

$$
R_{m, t}=\mu+\epsilon_{\mathrm{t}}
$$

where $E\left[\epsilon_{\mathfrak{t}} \mid \epsilon_{\mathrm{t}-1}, \epsilon_{\mathrm{t}-2}, \ldots, \epsilon_{1}\right]=0$ and $E\left[\left((1 / T) \sum_{t=1}^{T} E\left[\epsilon_{t}^{2} \mid \epsilon_{t-1}, \epsilon_{t-2}, \ldots, \epsilon_{1}\right]\right)\right] \rightarrow \sigma^{2}$ as $T \rightarrow \infty$ by assumption. Also, assume that $\sup _{t} E\left[\epsilon_{t}^{4}\right]<\infty{ }^{4}$

Let's consider the following $J$-period returns

$$
X_{t+J}(J)=\sum_{i=0}^{J-1} R_{m, t+J-i}, t=0, \ldots, T-J
$$

and the regression

$$
X_{t+J}(J)=\beta(J) Z_{t}(J)+\eta_{t+J}(J)
$$

where, in our case, $Z_{t}(J)$ is 1 . This case is not considered by Richardson and Stock (1989) but the analysis is similar.

The null hypothesis that we are interested in is $H_{0}: \beta(J)=0$. Testing whether $\beta(J)$ is zero requires constructing a standard error for $\beta(J)$. Asymptotic justification of the conventional computation of standard error in the ordinary least squares(OLS) estimator of $\beta(J)$ requires that the errors be serially uncorrelated. Consequently, one strategy is to define the sampling interval to be equal to the return interval, the $J$-period. In such non-overlapping samples, the number of observations can be small, even though the raw sample is large. However, if we use data sampled more finely than the forecast interval.

\footnotetext{
${ }^{4} B y$ assumption, the returns are allowed to be conditionally heteroskedastic. However, IGARCH process violates the assumption that $s u p_{t} E\left[\epsilon_{l}^{4}\right]<\infty$. See Engle and Bollerslev (1986).
} 
we need to consider the serial correlation of errors in estimation. Hansen and Hodrick (1980) proposed a variance estimator using specific weights on sample autocovariances of errors and regressors. The standard theory assumes that the overlap in the data (denoted by $J$ ) is fixed as $T \rightarrow \infty$ so that $J / T \rightarrow 0$. If we assume, as in Richardson and Stock (1989), that $J / T \rightarrow \delta$, then the conventional $t$-ratios of the coefficients in regression analysis have different asymptotic distributions. Under the assumption that $J / T \rightarrow \delta$, the OLS estimate of the coefficient of the regression (5),

$$
\hat{\beta}(J)=\frac{1}{T-J} \sum_{t=0}^{T-J} x_{t+J}(J)
$$

diverges as $T \rightarrow \infty$, and $\hat{\beta}(J) / \sqrt{T}$ has a limiting distribution given as a functional of Brownian motions. 5

Hansen and Hodrick's (1980) variance estimator of $\hat{\beta}(J)$ is computed as follows,

$$
\hat{w}^{2}(J)=\frac{1}{T} \sum_{i=-(J-1)}^{J-1} \frac{T-|i|}{T} \hat{\gamma}_{u}^{2}(i)
$$

where

$$
\hat{\gamma}_{u}^{2}(i)=\frac{1}{T-J-|i|} \sum_{i=\{i \mid}^{T-J}\left(x_{i+J}(J)-\dot{\beta}(J)\right)\left(x_{i+J-|i|}(J)-\hat{\beta}(J)\right)
$$

It also converges to a functional of Brownian motion, as $T \rightarrow \infty$ and $J / T \rightarrow \delta .^{6}$

\footnotetext{
${ }^{5}$ See Appendix A

${ }^{6}$ See Appendix A
} 
Table 1: Distribution of the $t$-ratio statistic (6,000 Monte Carlo simulations).

\begin{tabular}{|l|rrrrrrr|}
\hline & \multicolumn{7}{|c|}{ Percentile } \\
& $2.5 \%$ & $5 \%$ & $10 \%$ & $50 \%$ & $90 \%$ & $95 \%$ & $97.5 \%$ \\
\hline$\delta=1 / 200$ & -1.99 & -1.67 & -1.26 & 0.02 & 1.32 & 1.70 & 2.04 \\
$\delta=1 / 100$ & -2.02 & -1.69 & -1.28 & 0.01 & 1.34 & 1.74 & 2.08 \\
$\delta=1 / 40$ & -2.10 & -1.77 & -1.34 & 0.02 & 1.37 & 1.78 & 2.17 \\
$\delta=1 / 20$ & -2.34 & -1.91 & -1.45 & 0.03 & 1.48 & 1.96 & 2.40 \\
\hline
\end{tabular}

Theorem 3.1 Under the assumptions in Phillips (1987), conventional t-ratio, $t_{\dot{\beta}}$, converges, not to normality, but to a functional of Brownian motion, as $T \rightarrow \infty$ and $J / T \rightarrow \delta$,

$$
t_{\hat{\beta}}=\frac{\hat{\beta}(J)}{\sqrt{\hat{w}^{2}(J)}} \Longrightarrow \frac{\frac{1}{1-\delta} \int_{0}^{1-\delta}\{W(s+\delta)-W(s)\} d s}{\left[\int_{-\delta}^{\delta} \Gamma_{u}(\rho, \delta) d \rho\right]^{1 / 2}}
$$

Since the distribution of $t_{\hat{\mathcal{A}}}$ does not follow the student $t$-distribution, we compute the critical values through Monte Carlo simulation as shown in Table 1. These can be used to test hypotheses for different values of $\delta=J / T$ and will give better finite sample critical values.

\section{Estimation of the Value of Variance Forecasts with a} Simulated Market in Index-Options of Various Maturities

The objective is to estimate the potential profit from improving the variance forecast when pricing options of various maturities. By using a simulated option market instead 
of observing actual index-option prices, we are able to circumvent perennial difficulties that plague empirical studies of option prices: non-synchroneity of option and stockprices, market depth, the wild card delivery option, and dividend protection as well as limited maturity dates and strikes. Thus, with a simulated index-option market, the Black-Scholes model need only be reconciled with the fact that variance is stochastic.

Table 2 shows average straddle prices that were written every day between 1968 - 1991 on one dollar's worth of the NYSE index, with an exercise price of $\$ 1$ plus the risk-free rate (for the life of the option). These average prices are categorized by maturity (in calendar days) for the alternative forecasting methods: MA, GARCH, GARCH-I, GARCH-D and GARCH-DI. The interesting feature of these prices is that GARCH prices are lower than MA prices for maturities shorter than three months and GARCH prices are greater than MA prices for maturities longer than nine months. For maturities up to 50 days, integration over alternative GARCH forecasts shown by the GARCH-I column, produced higher average straddle prices than the GARCH. However, for maturities in the range of 60 - 365 days, integration produced smaller average straddle prices. Finally, adjusting GARCH and GARCH-I variance forecasts to account for weekends increases average straddle prices for maturities up to 14 days and decreases average straddle prices for maturities greater than 14 days, compared to GARCH prices.

Table 3 shows the annualized rate of return from a daily investment in straddles on the NYSE index by two hypothetical agents one using MA and the other using GARCH variance forecasts. Each day, each agent invests one dollar in each maturity (1 to 365 days) of straddles. The transaction price is the average of the reservation prices of the agents based on their private variance forecasts. The position in each straddle is held 
to maturity, and the agent who takes the short position in a given maturity (due to a lower variance forecast), invests the $\$ 1$ proceeds from writing the straddle plus the $\$ 1$ endowment in the NYSE-index portfoliio for the life of the straddle. Upon maturity each position is settled according to the actual level of the NYSE index on the maturity date. Because the agents trade every day straddles of various maturities, holding periods overlap. The straddles are always written on one dollar's worth of the NYSE index, with an exercise price of $\$\left(1+r_{f}\right)$, where the risk-free rate is taken from the one month T-bill yields. The trades are executed at the average of the variance-forecast driven reservation prices of the two agents.

The first column of Table 3 shows the maturity of the traded straddles; there are 5,921 observations for each maturity. The third column in Table 3 shows the time weighted annualized rate of return to the GARCH agent from the cash flows received from, and paid to, the MA agent in lieu of settlements of the maturing straddles - based on the history of the NYSE index over the period 1968-1991. The fourth column and fifth column in Table 3 enumerate the annualized rate of return to the MA and the GARCH agents from investing in the NYSE-index portfolio on days when they took short positions. The sixth and seventh columns shows the total annualized rate of return of the MA and the GARCH agents from the entire trading activity. Because the investment in the NYSE index augments the short positions, the competition between the agents is not a zero sum game.

The Hansen-Hodrick standard errors are given in parenthesis next to average rate of returns and in the seventh column ordinary standard errors are given in the same parenthesis as well. The difference between these two standard errors is growing as the maturity increases. For 1-year option, the Hansen-Hodrick standard error is ten times 
greater than the ordinary standard error! The eighth column compute t-ratios for the total annualized rates of return of the GARCH agents in the seventh column using the Hansen-Hodrick standard errors. Those that are significant at $5 \%$ can be chosen by the critical values of the Table 1 in the previous section.

Table 3 shows enormous rates of return to the GARCH agent (at the expense of the MA agent) from trades of short-term options: $1,903 \%$ for one-day options. The rates of return are monotone decreasing with maturity up to 90 days and then increase again. While at 22 -days maturity the $126 \%$ annualized return is statistically and economically significant, it is obvious that the apparent advantage of the GARCH forecasts over the MA forecasts is fading with the forecast horizon. Above 22-days maturity, the average rate of return is an economically significant $100 \%$ at maturity of 24 days, but is no longer statistically significant. Note that the average rates of return to the GARCH agents are positive for all maturities, and are still at $29 \%$ for a 1 -year maturity.

The return from investing short position proceeds in the market are not too different for both agents, indicating that none specialized in short positions. While the higher average straddle price (up to 90-days) from MA variance forecasts (Table 2) would have us expect that the MA agent would take less short positions, proceeds from investing short positions show that the variance in straddle prices swamps the mean difference between the methods, and that the MA and GARCH agents' short/long psitions are similarly distributed. The bottom line is that the GARCH forecasts are economically, and statistically, significantly better than the MA forecasts in pricing options of up to 22 days maturity, for which the GARCH agent earned an average return of $126 \%$ from the MA agent.

Tables $4-6$ present results from repeating the straddle trading exercise replacing 
the GARCH agents of Table 3 with improved GARCH forecasts. Table 4 presents the results from integrating the straddle price from the Black-Scholes formula over the GARCH forecasts based on the distribution of forecasting errors from past forecasts to implement the Hull and White correction. The rates of return earned by the GARCH-I agent are markedly better than those earned from the MA agent by the GARCH agent. In Table 5 GARCH-D agent accounts for the weekend effect by using equation (3). The rates of return earned by the GARCH-D agent for short maturities are better than those by the GARCH agent from MA agent. The improvement in the forecasts fades with maturity, and becomes insignificant for maturities over 20 days. Tables 6 shows the results from incorporating the weekend effect into the integrated GARCH forecasts. The average annualized return for a 28 -days maturity is $112 \%$ (compared with $59 \%$ for the GARCH agent), and is still statistically significant.

Figure 2 plots the cumulative percentage returns to the GARCH and GARCH-DI agents from the MA agent by maturity. The graphs show the economically significant improvement that can be obtained by integrating the forecast with the Hull-White method and accounting for the weekend effect.

Tables $7-9$ measure the value of the improved GARCH forecasts by trading against a GARCH agent. Tables 7 shows that the GARCH-I forecasts are economically and statistically better than the GARCH forecasts for up to 90 -days maturity. Incorporating both improvements yields better rates of return for almost all naturities.

\section{Conclusions}

The return history of the NYSE suggests that the choice of variance forecasts for pricing index options can make a significant economic difference to investors. The impact on 
investment in index options from choosing between different one-day forecast methods used in this paper apply to options of maturity of up to one month. An important step in improving variance forecasts is to make Hull and White's (1987) modification to Black and Scholes's (1973) formula. Moreover, incorporating the weekend effect into the parameter estimation and forecast specification is also important.

Using simulated option markets has circumvented the problems of market depth. price synchroneity, dividend protection and delivery options. Thus these results are not subject to objections grounded in problems that plague empirical work with actual option prices. The stage is now set to investigate actual option prices and attempt to isolate price events that result from changing variance forecasts from those that result from one of the market imperfections that traders are facing. 


\section{A Appendix}

Proof of Theorem 3.1. Let

$$
S_{\tau}=\sum_{i=1}^{T} \epsilon_{t}, \tau=1,2, \ldots, T,
$$

and set $S_{0}=0$. The functional central limit theorem(FCLT $)^{7}$ states that the random function $\left\{(1 / \sigma \sqrt{T}) S_{[T \lambda]}\right\}, \lambda \in[0,1]$, weakly converges to a standard Brownian motion process on the unit interval, $W(\lambda)$ : as $T \rightarrow \infty$,

$$
\frac{1}{\sigma \sqrt{T}} S_{[T \lambda]} \Longrightarrow W(\lambda)
$$

And by the continuous mapping theorem, we can derive the followings, as $T \rightarrow \infty$,

$$
T^{-3 / 2} \frac{1}{\sigma} \sum_{t=1}^{T} S_{[t \lambda]} \Longrightarrow \int_{0}^{\lambda} W(s) d s
$$

and

$$
T^{-2} \frac{1}{\sigma^{2}} \sum_{t=1}^{T} S_{[t \lambda]}^{2} \Longrightarrow \int_{0}^{\lambda} W(s)^{2} d s .
$$

Since the $J$-period return was defined as $X_{t+J}(J)=\sum_{i=0}^{J-1} R_{m, t+J-i}, t=0, \ldots, T-$

\footnotetext{
${ }^{7}$ See Billingsley (1968) or Hall and Heyde (1980) or Herndorf (1984) for functional central limit theorem and continuous mapping theorem. For applications of the FCLT and continuous mapping theorem, see Phillips (198i).
} 
$J$, the OLS estimate of $\hat{\beta}(J)$ of the regression (5) in section 3 can be rewritten as follows,

$$
\begin{aligned}
\hat{\beta}(J) & =\frac{1}{T-J} \sum_{i=0}^{T-J} x_{i+J}(J) \\
& =\frac{1}{T-J} \sum_{i=0}^{T-J}\left\{\sum_{i=0}^{i+J} R_{m, i}-\sum_{i=0}^{i} R_{m, i}\right\} .
\end{aligned}
$$

Using equation (10) and equation (11), the following holds, as $T \rightarrow \infty$ and $J / T \rightarrow \delta$.

$$
\frac{1}{\sqrt{T}} \hat{\beta}(J) \Longrightarrow \sigma \frac{1}{1-\delta} \int_{0}^{1-\delta}\{W(s+\delta)-W(s)\} d s
$$

Also, using equation (10), equation (11) and equation (12), the following holds, as $T \rightarrow \infty, J / T \rightarrow \delta$ and $i / T \rightarrow \rho$,

$$
\begin{aligned}
\frac{1}{T} \hat{\gamma}_{u}^{2}(i) & =\frac{1}{T} \frac{1}{T-J-|i|} \sum_{i=|i|}^{T-J}\left(x_{i+J}(J)-\hat{\beta}(J)\right)\left(x_{i+J-|i|}(J)-\hat{\beta}(J)\right) \\
& \Longrightarrow \sigma^{2}(1-|\rho|) \frac{1}{1-\delta-|\rho|} \int_{|\rho|}^{1-\delta} U_{\delta}(s+\delta) U_{\delta}(s+\delta-|\rho|) d s
\end{aligned}
$$

where $U_{\delta}(\lambda)$ is defined as follows,

$$
U_{\delta}(\lambda)=\{W(\lambda)-W(\lambda-\delta)\}-\frac{1}{1-\delta} \int_{0}^{1-\delta}\{W(\lambda)-W(\lambda-\delta)\} d \lambda
$$

Hence, Hansen and Hodrick's (1980) variance estimator of $\hat{\beta}(J)$ converges to a functional of Brownian motion, as $T-\propto$ and $J / T \rightarrow \delta$,

$$
\begin{aligned}
\frac{1}{T} \hat{w}^{2}(J) & =\frac{1}{T^{2}} \sum_{i=-(J-1)}^{J-1} \frac{T-|i|}{T} \hat{\gamma}_{u}^{2}(i) \\
& \Longrightarrow \sigma^{2} \int_{-\delta}^{\delta} \Gamma_{u}(\rho, \delta) d \rho
\end{aligned}
$$


where $\Gamma_{u}(\rho, \delta)$ is defined as follows,

$$
\Gamma_{u}(\rho, \delta)=(1-|\rho|) \frac{1}{1-\delta-|\rho|} \int_{|\rho|}^{1-\delta} U_{\delta}(s+\delta) U_{\delta}(s+\delta-|\rho|) d s
$$

Therefore, conventional t-ratio, $t_{3}$, converges to a functional of Brownian motion, as $T \rightarrow \infty$ and $J / T \rightarrow \delta$,

$$
\begin{aligned}
t_{\dot{\beta}} & =\frac{\dot{\beta}(J)}{\sqrt{\hat{w}^{2}(J)}} \\
& \Rightarrow \frac{\frac{1}{1-\delta} \int_{0}^{1-\delta}\{W(s+\delta)-W(s)\} d s}{\left[\int_{-\delta}^{\delta} \Gamma_{u}(\rho, \delta) d \rho\right]^{1 / 2}}
\end{aligned}
$$




\section{References}

Billingsley, P. 1968. Convergence of probability measure. New York, NY: Wiley.

Black, F., and M. S. Scholes. 1973. The pricing of options and corporate liabilities. Journal of Political Economy 81:637-659.

Bollerslev, T., R. Chou, and K. F. Kroner. 1992. ARCH modelling in finance: A review of the theory and empirical evidence. Journal of Econometrics 52:5-59.

Brenner, M., and D. Galai. 1986. Implied interest rates. Journal of Business 59(3):493-508.

Engle, R. F. 1982. Autoregressive conditional heteroskedasticity with estimates of the variance of U.K. inflation. Econometrica 50:987-1008.

Engle, R. F., and T. Bollerslev. 1986. Modelling the persistence of conditional variances. Econometric Reviews 5:1-50;81-87.

Engle, R. F., T. Hong, A. Kane, and J. Noh. 1993. Arbitrage valuation of variance forecasts. Adaunced Futures and Options Research 6:393-415.

French, K. G., and R. Roll. 1986. Stock return variance: the arrival of information and the reaction of traders. Journal of Financial Economics 17(1):5-26.

Hall, P., and C. C. Heyde. 1980. Martingale limit theory and its application. New York, NY: Academic Press.

Hansen, L. P., and R. J. Hodrick. 1980. Forward exchange rates as optimal predictors of future spot rates: An econometric analysis. Journal of Political Economy 88:829853.

Herndorff, N. 1984. A functional central limit theorem for weakly dependent sequences of random variables. Annals of Probability 12:141-153.

Heston, J. 1992. Option pricing with stochastic volatility. Working paper, Yale University.

Hull, J, and A. White. 1987. The pricing of options on assets with stochastic volatility. Journal of Finance 42:281-300.

McDonald, R. L., and D. Siegel. 1984. Option pricing when the underlying asset earns a below-equilibrium rate of return. Journal of Finance 39:261-265.

Phillips, P. C. B. 1987. Times series regression with a unit root. Econometrica $55: 227-301$. 
Richardson, M., and J. H. Stock. 1989. Drawing inferences from statistics based on multiyear asset returns. Journal of Financial Economics 25:323-348.

Schmalensee, R., and R. R. Trippi. 1978. Common stock volatility expectations implied by option prices. Journal of Finance 33:129-147. 
Figure 1: Option Prices.

(June 20,1968-December 31, 1991)

30-days straddles

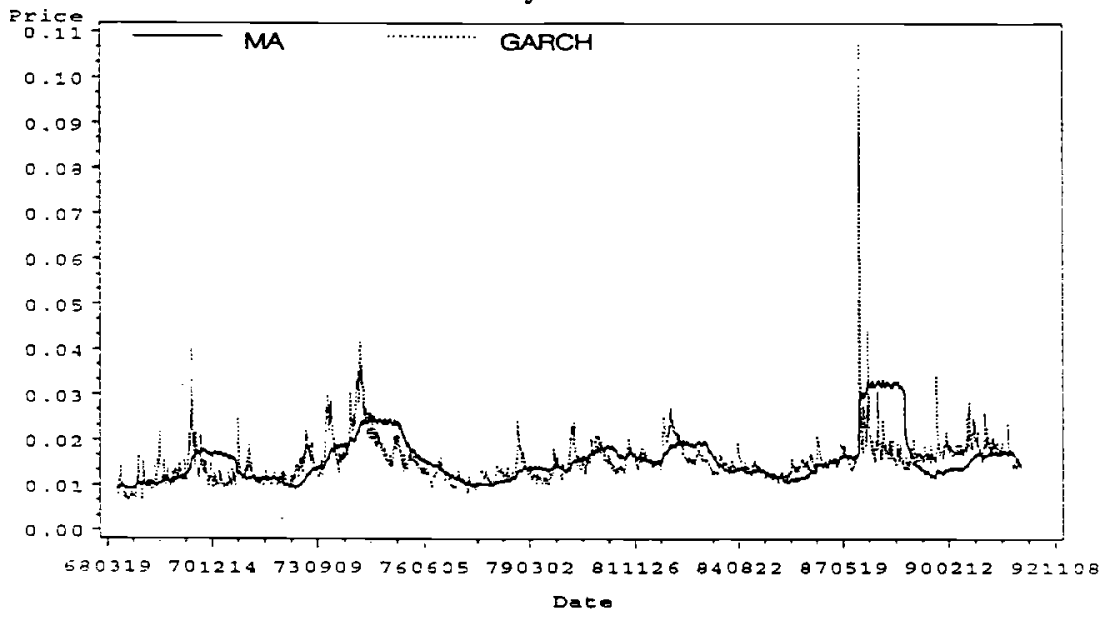

36.5-days straddles

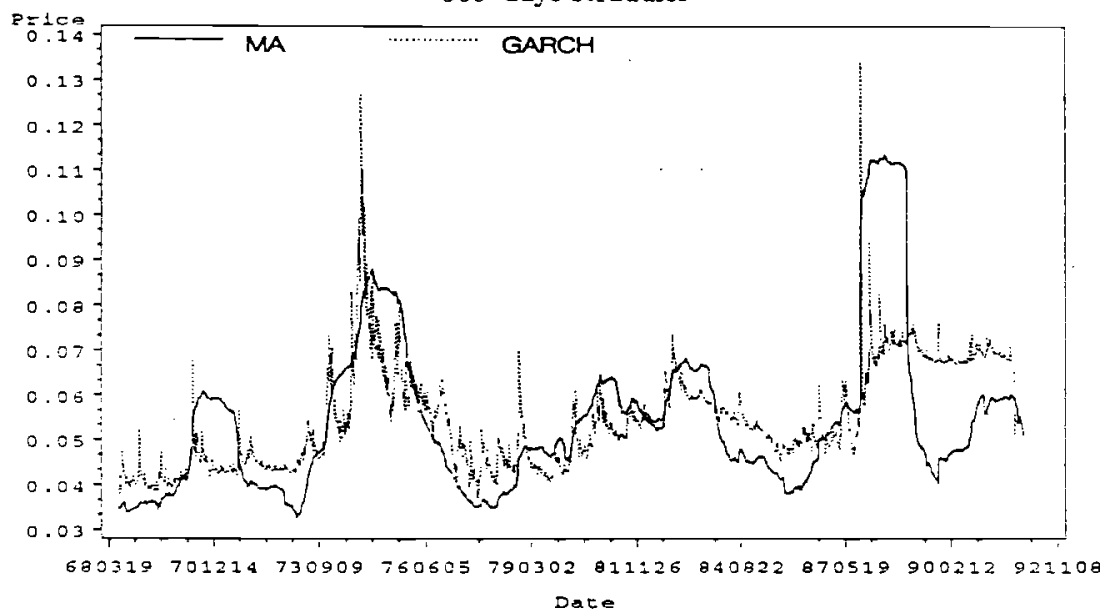


Figure 2: Cumulative Profits(over MA).

(June 20,1968 - December 31, 1991)

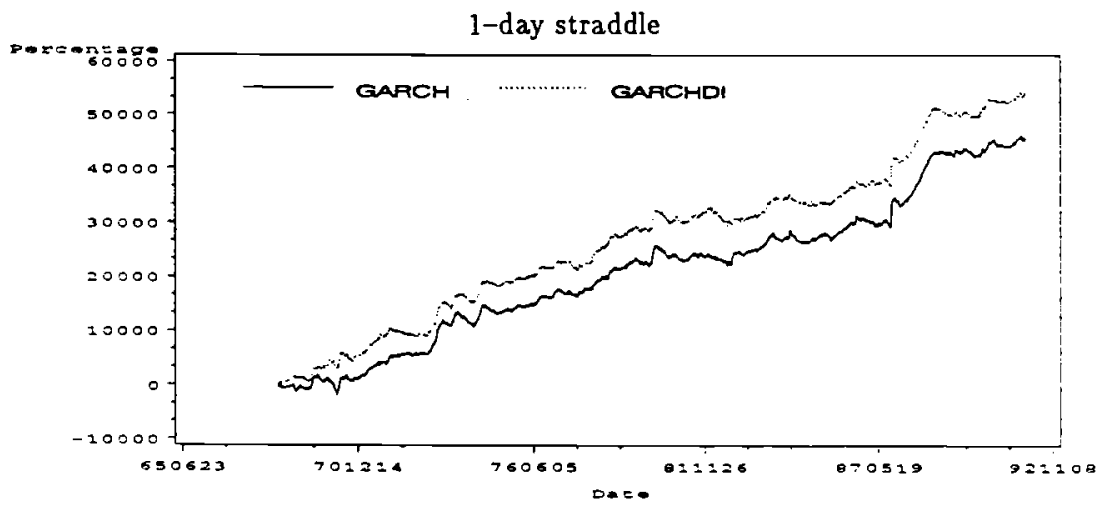

30-days straddle

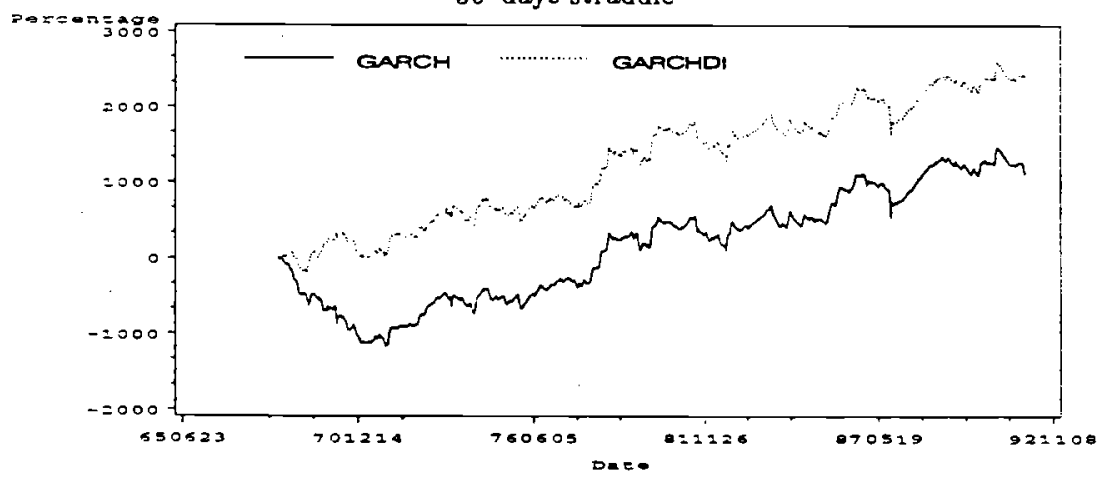

\section{5-days straddle}

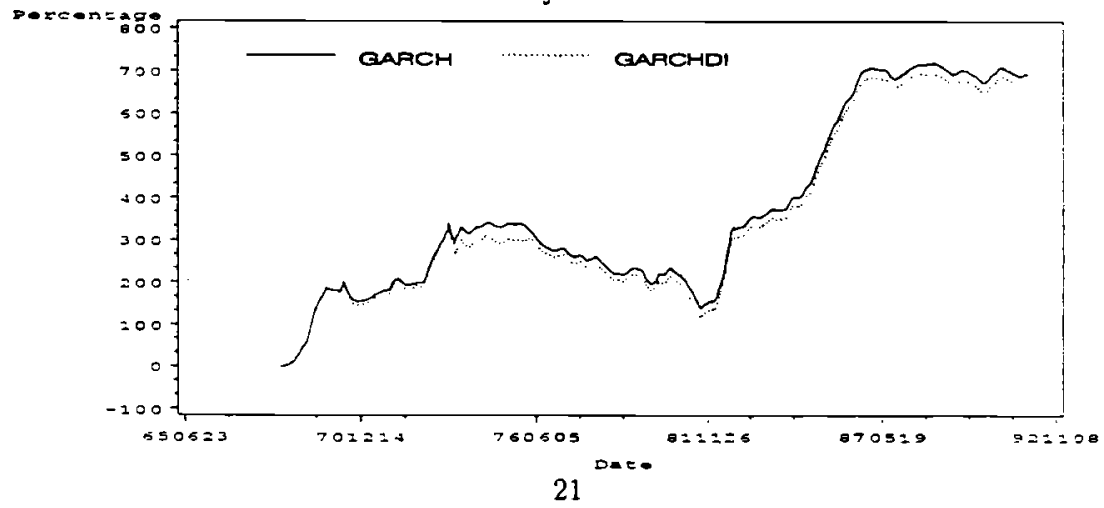




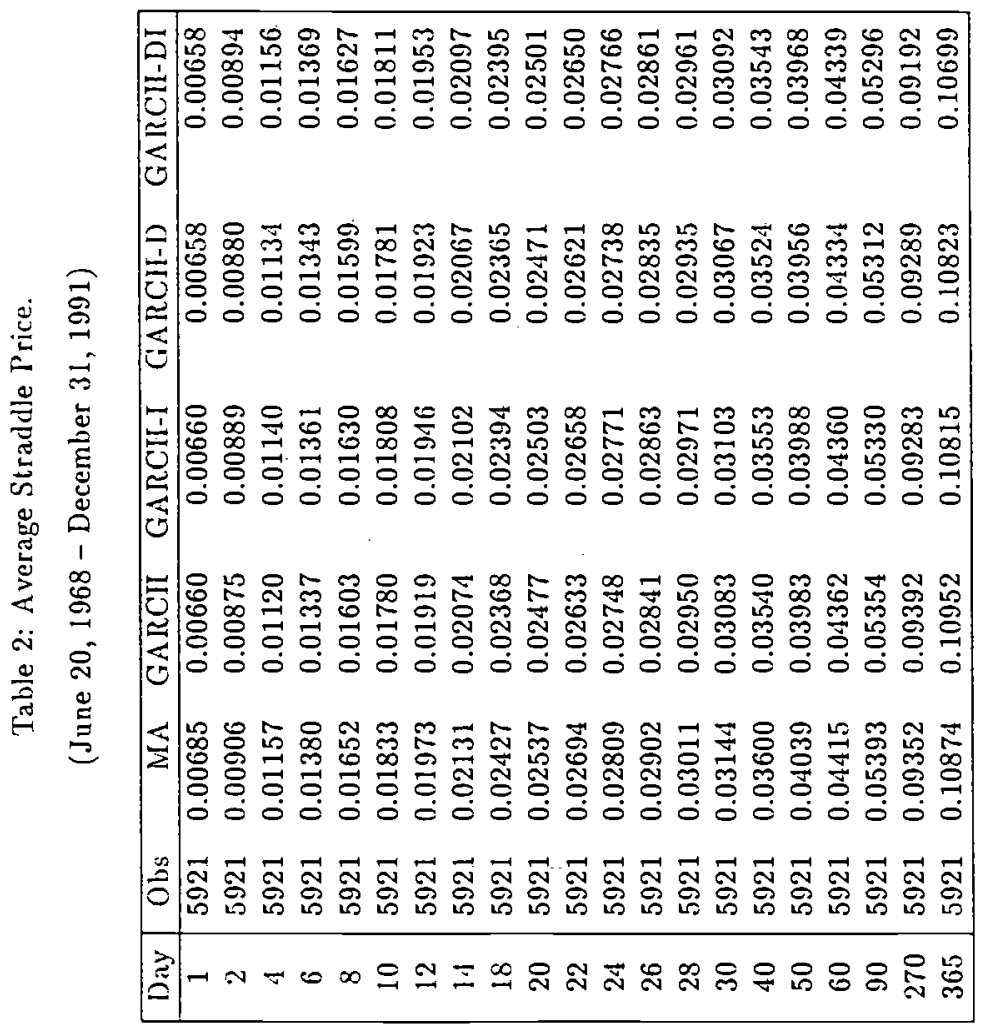




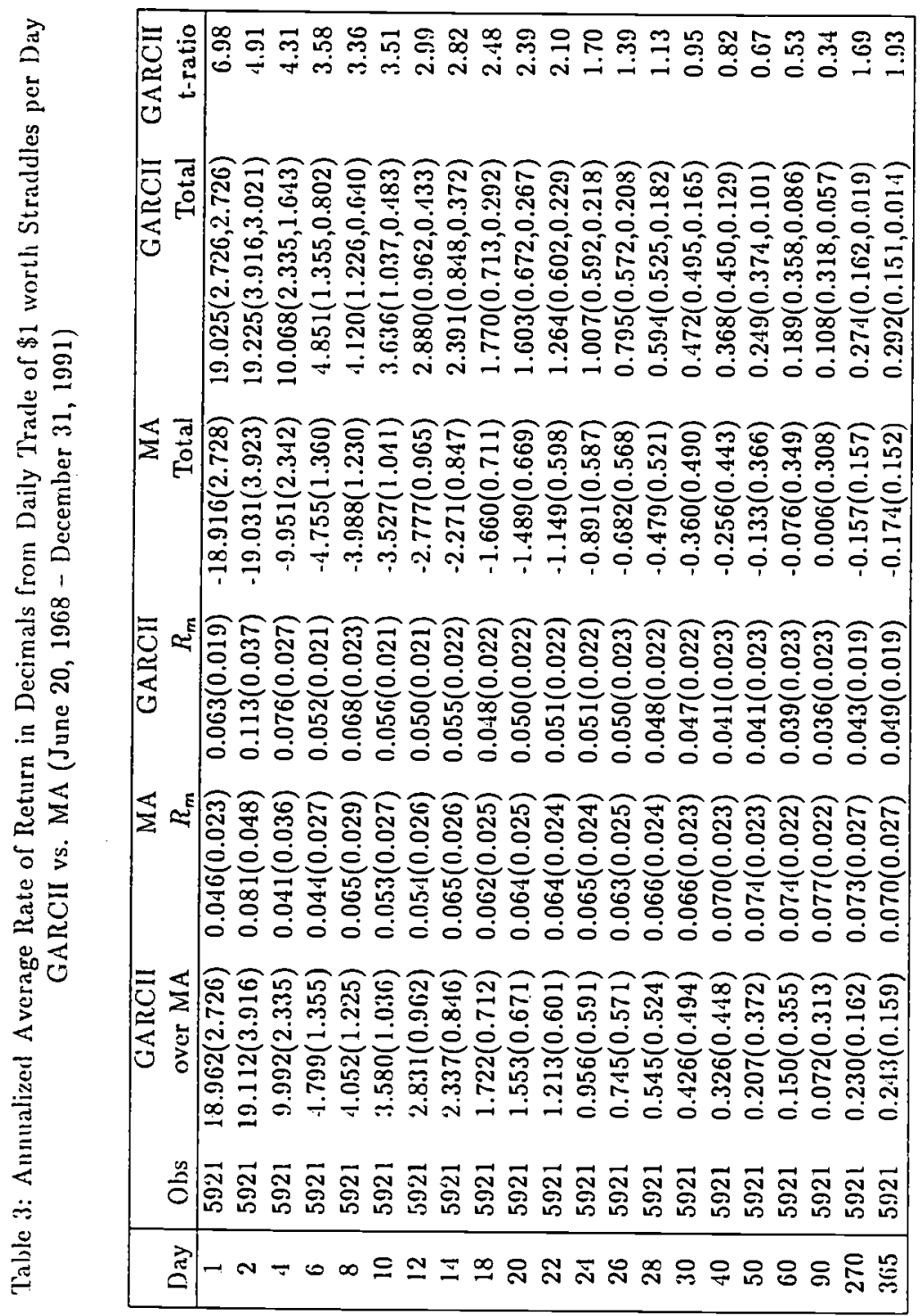




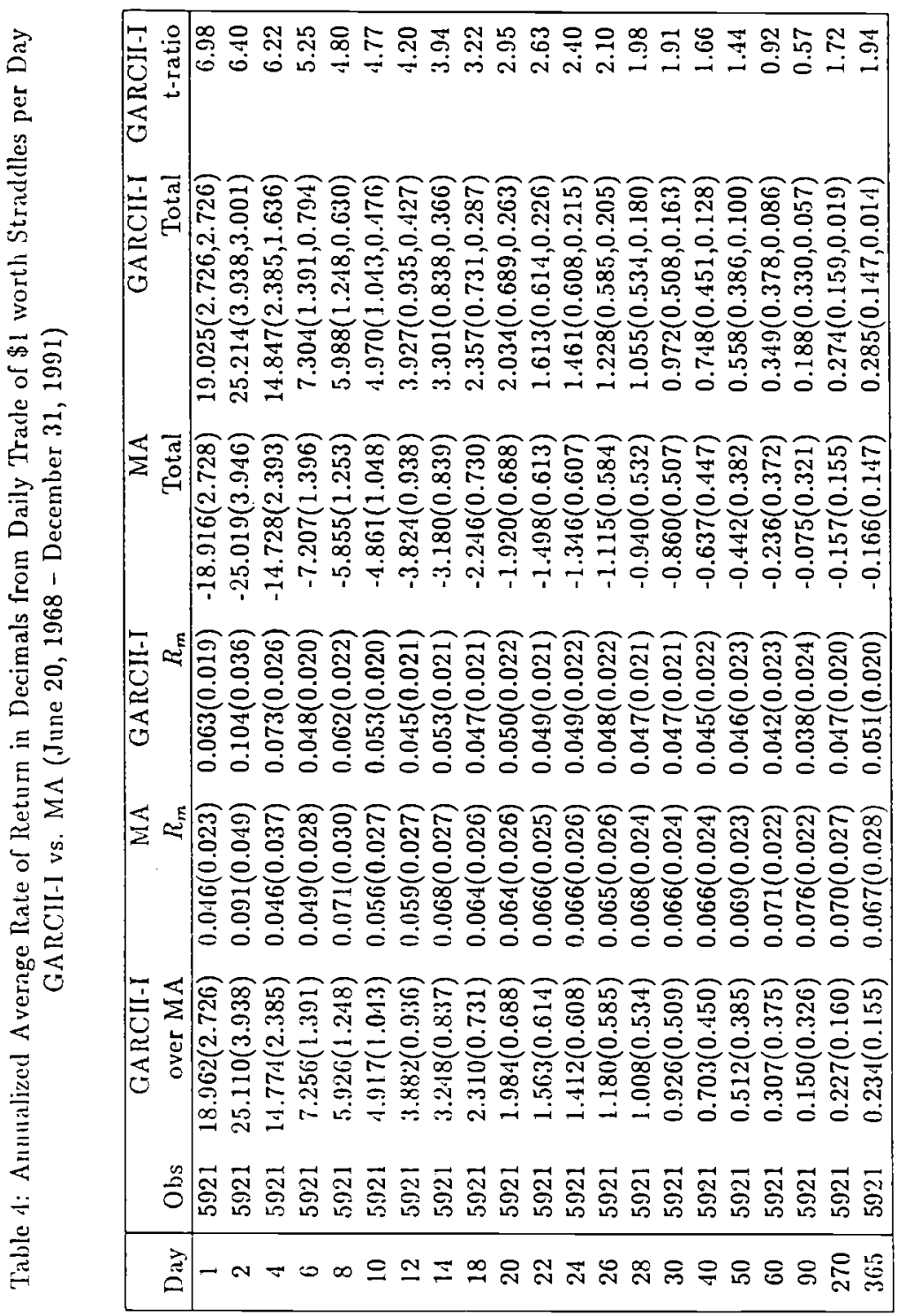




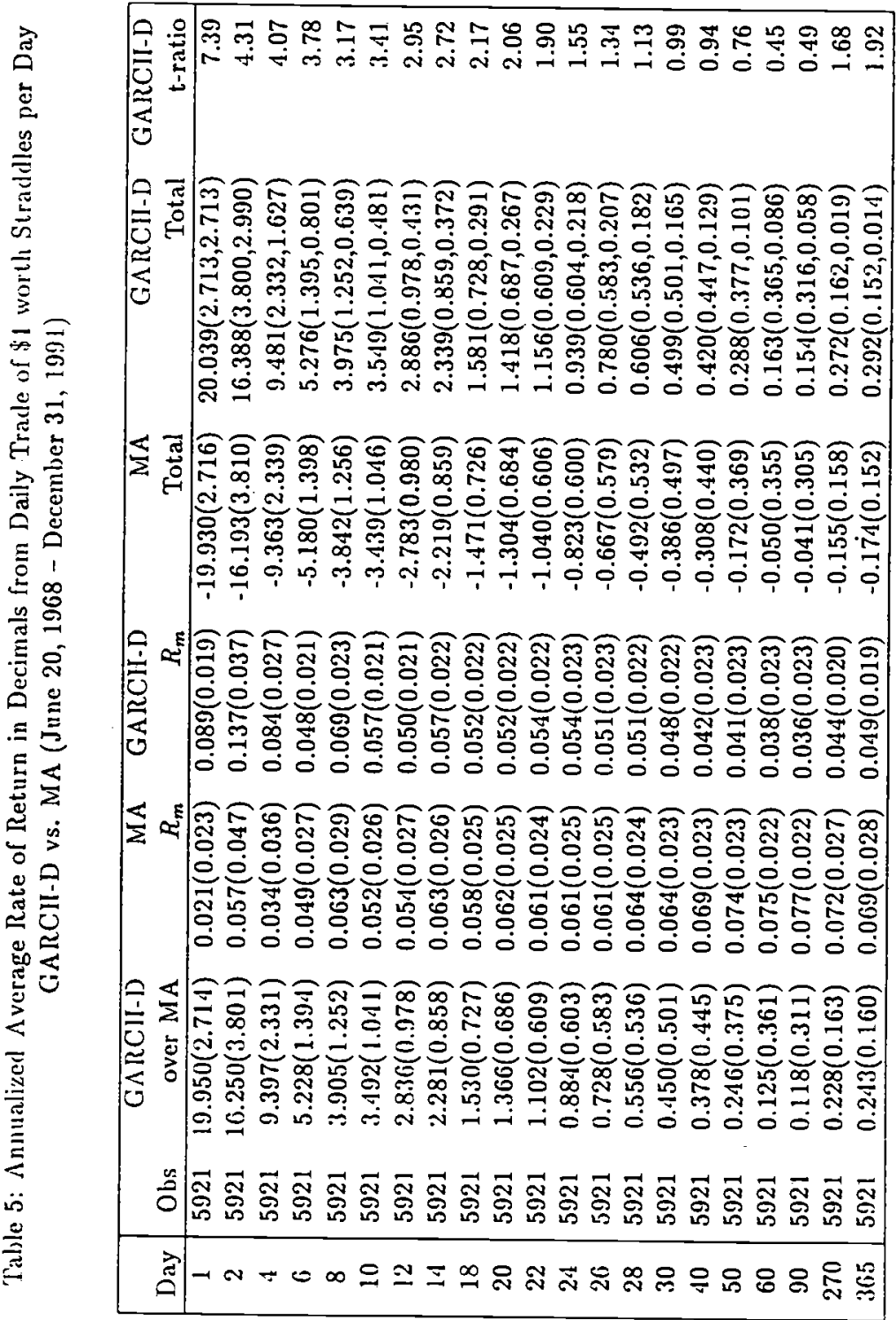




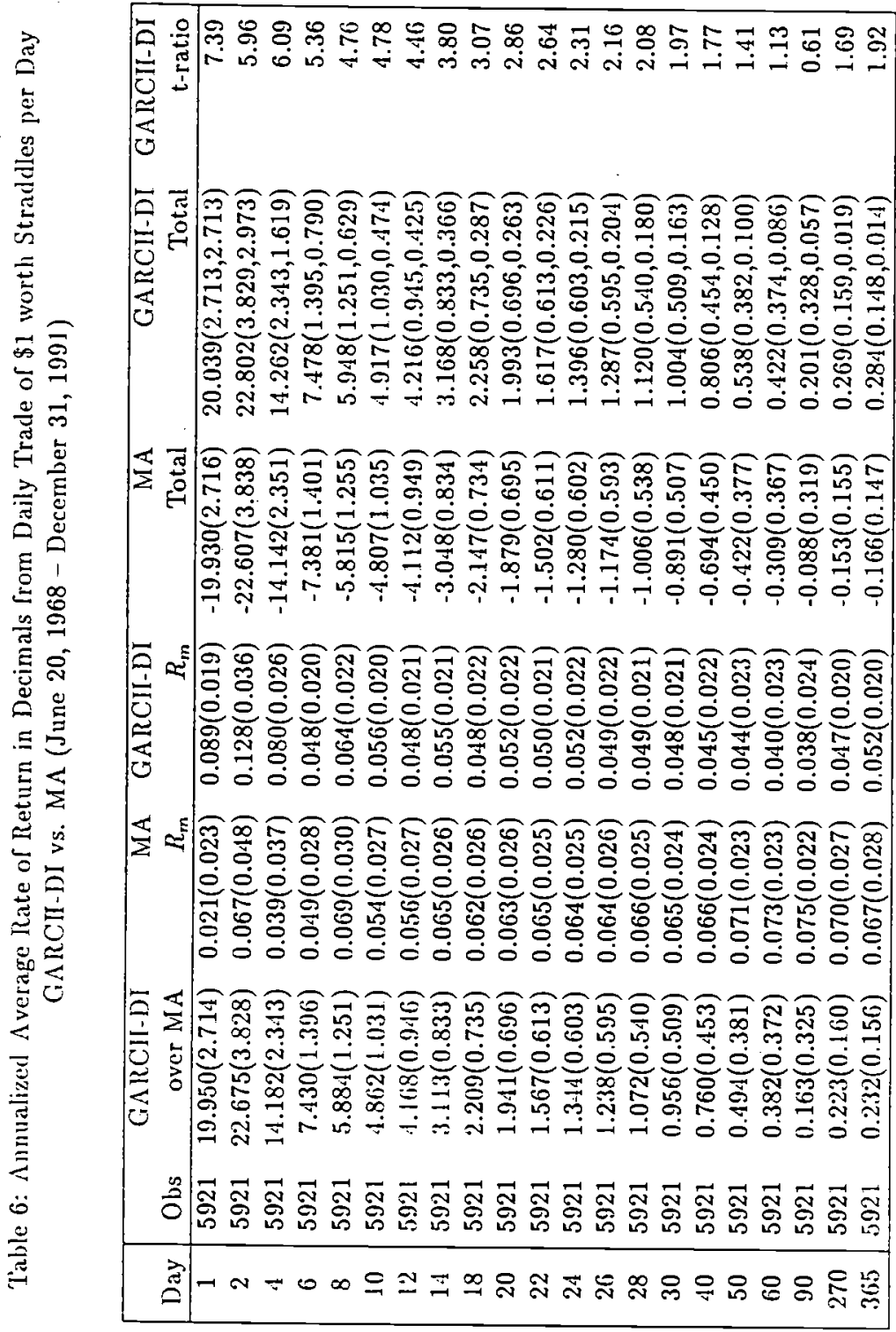




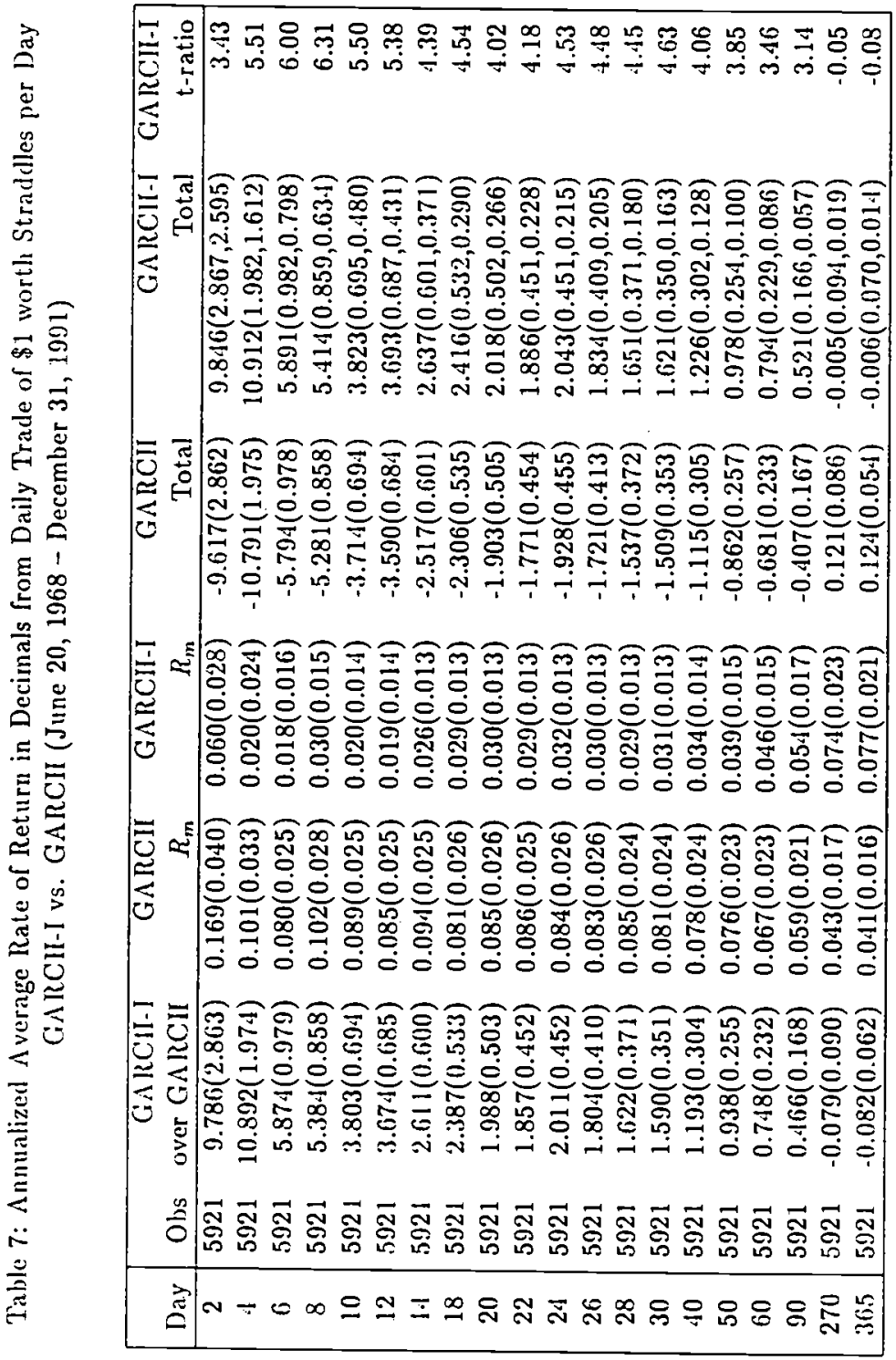




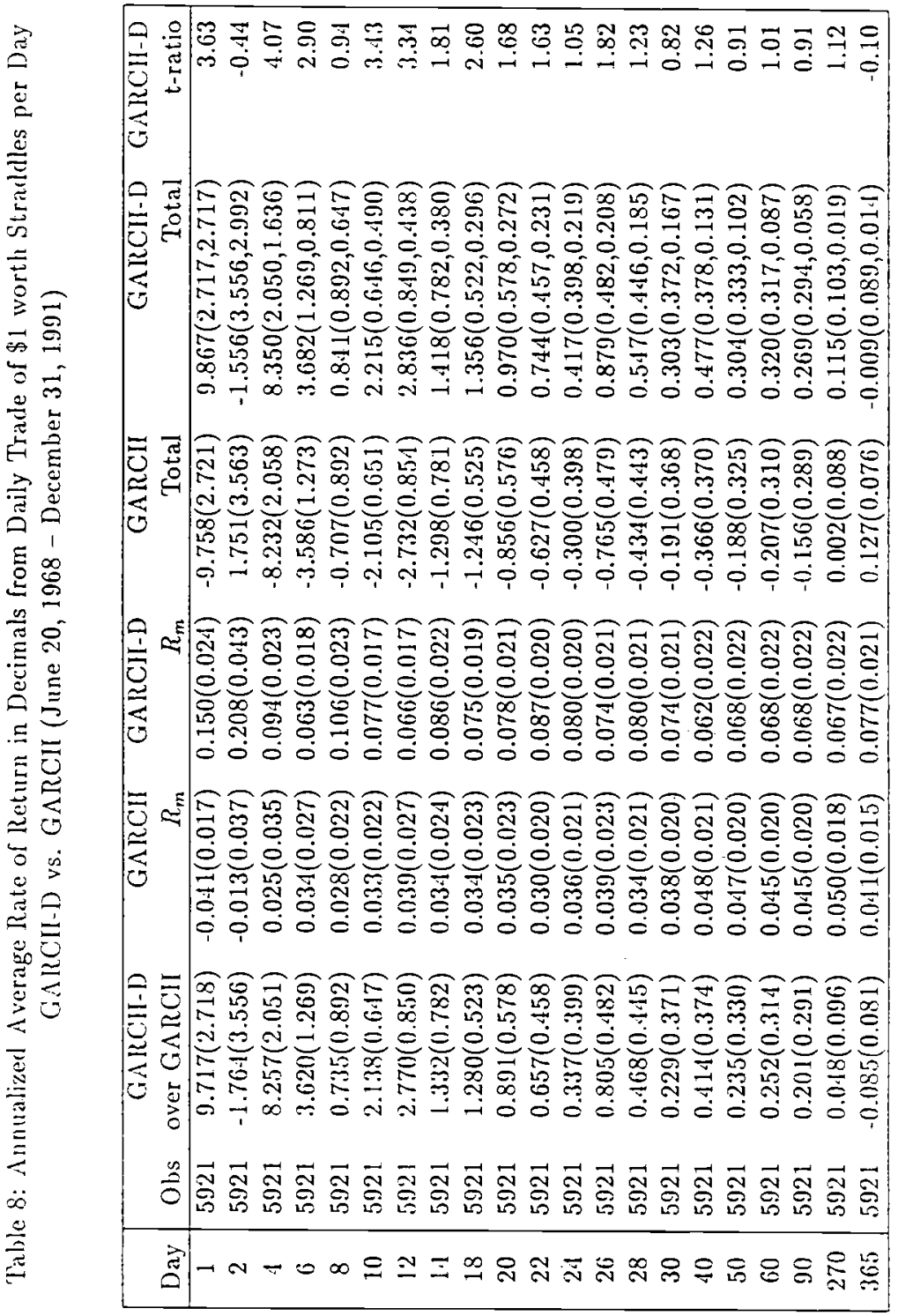




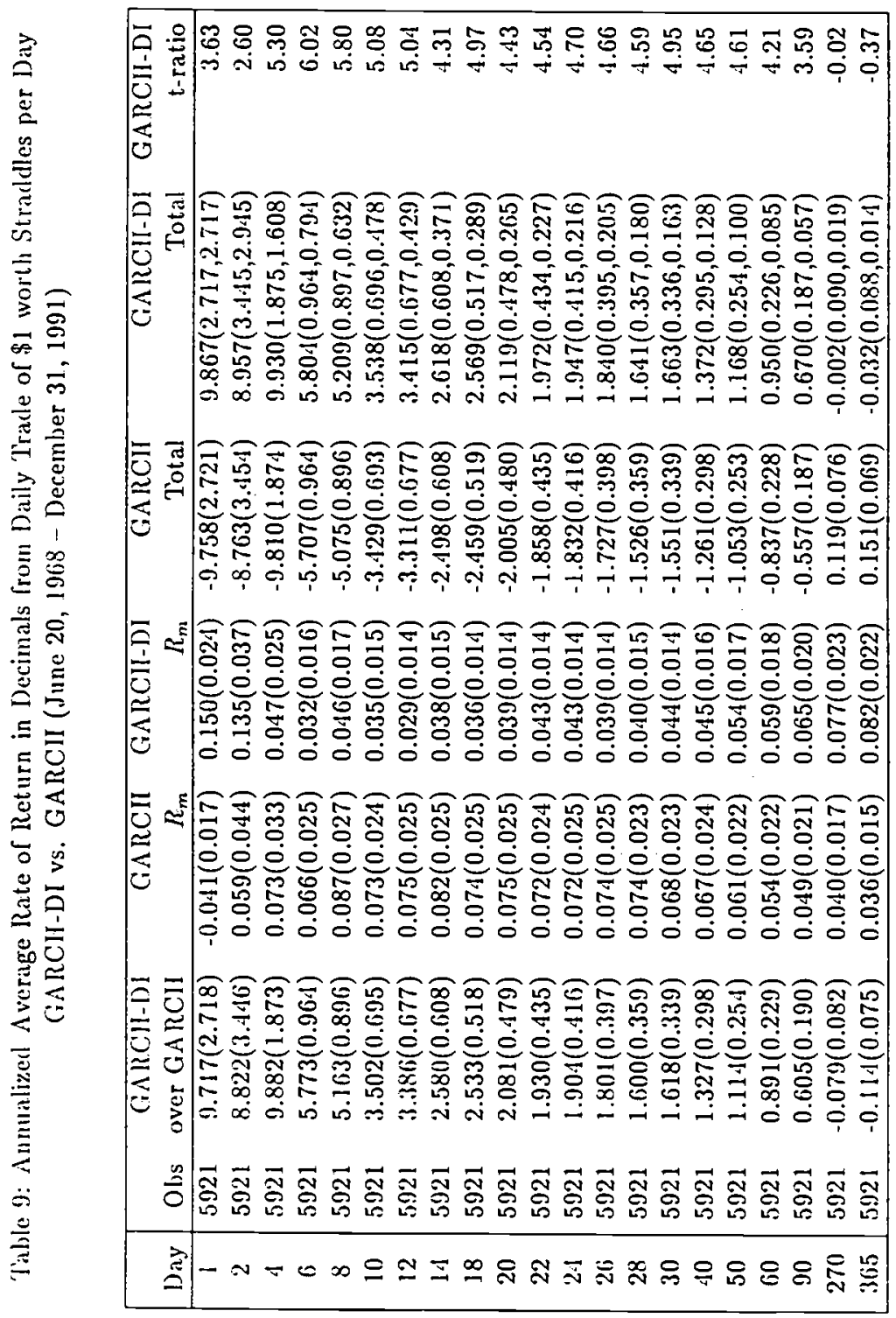

